\title{
Augmented binary multi-labeled CNN for practical facial attribute classification
}

\author{
Mohammed Berrahal, Mostafa Azizi \\ MATSI Research Lab, ESTO, Mohammed First University, Oujda, Morocco
}

\begin{tabular}{l} 
Article Info \\
\hline Article history: \\
Received Mar 21, 2021 \\
Revised May 23, 2021 \\
Accepted Jun 1, 2021 \\
\hline
\end{tabular}

\section{Keywords:}

CNN

Data augmentation

Deep learning

Face attributes

Face sketch image

Image classification

Multi-label learning

\begin{abstract}
Both human face recognition and generation by machines are currently an active area of computer vision, drawing curiosity of researchers, capable of performing amazing image analysis, and producing applications in multiple domains. In this paper, we propose a new approach for face attributes classification (FAC) taking advantage from both binary classification and data augmentation. With binary classification we can reach high prediction scores, while augmented data prevent overfitting and overcome the lack of data for sketched photos. Our approach, named Augmented binary multilabel CNN (ABM-CNN), consists of three steps: i) splitting data; ii) transformed-it to sketch (simplification process); iii) train separately each attribute with two convolutional neural networks; the whole process includes two networks: the first (resp. the second) one is to predict attributes on real images (resp. sketches) as inputs. Through experimentation, we figure out that some attributes give high prediction rates with sketches rather than with real images. On the other hand, we build a new face dataset, more consistent and complete, by generating images using Style-GAN model, to which we apply our method for extracting face attributes. As results, our proposal demonstrates more performances compared to those of related works.
\end{abstract}

This is an open access article under the CC BY-SA license.

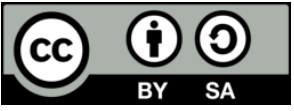

\section{Corresponding Author:}

Mohammed Berrahal

Superior School of Technology Oujda (ESTO), MATSI Research Lab

Mohammed First University, Oujda, Morocco

Email:m.berrahal@ump.ac.ma

\section{INTRODUCTION}

In just the past few years, artificial intelligence has taken the world by surprise, diving rapid progress especially in the field of deep learning, FAC and pattern recognition problem have seen tremendous progress. This contributes and plays an important role in security [1], [2] like access control for PCs or smartphone, video surveillance, criminal authentication, face sketch [3] and face photo for the law enforcement application [4]. The main property of FAC [5] is to predict multiple face features, state and emotion [6] on the given image or face portrait. Various algorithms have reached an excellent result on multiple levels for FAC, either apply directly CNN [7] models to extract face features, or using methods for improving the learning by distributing the attributes into two categories: objective attributes like wearing a hat, eyeglasses, bangs and subjective ones like smiling, big lips [8]. Some methods focus on grouping some attributes on the basis of their intercorrelations [9], while others target detecting face landmark localization [10] to reduce the noise.

Despite their wide application, FAC remains a challenge for researchers. There is still difficulty to recognize different face attributes on a giving image and it may require more attentions to cover different regions of the face, and a lack of large datasets with heterogeneous face images and sketches, or any 
additional information that can help to increase our accuracy. In this paper, we present a new augmented binary multi-label CNN-based method (ABM-CNN) to deal with different aspects of FAC for real facial images and sketches. Initially, we transform our multi-label problem to a binary problem. To this end, we develop an algorithm to transform our problem to mini-problems of binary classification by splitting the dataset of every attribute to two classes $(0$ or 1$)$ if the attribute exists in the image, then affected 1 , and 0 if no. After that we perform data augmentation to multiply data for solid learning, we convert every image in the dataset into 9 images by changing its perspectives. Due to the lack of sketches, we decide to transform our data to sketches. We run our CNN model for both real images and sketches, then we combine the prediction results for each attribute. Our prediction module in ABM-CNN outputs 40 facial attributes such as hair color, gender identification, smiling, attraction, and hat or glass wearing. On the other hand, we create our own dataset based on generated images using the style-GAN model [11], and we use it to create generated faces. Finally, we apply our ABM-CNN model to extract attributes and save it in a CSV file, to serve our next research in computer vision. The rest of this article is formulated as follows: In the second section, we recall backgrounds about convolutional neural network (CNN), data augmentation, binary classification and multi-label learning. In the third section, we discuss the related works. We present our implementation in the fourth section. Before concluding, the fifth section summarizes our results, and compares them with previous works.

\section{BACKGROUND}

\subsection{Convolutional neural network $(\mathrm{CNN})$}

One of the best learning algorithms the understanding image content, the CNN is a feedforward multilayered hierarchical network, the layers are using multiple convolutional kernels to transform the given correlated data, for extracting useful features from them, in the other hand the output of the convolutional kernels is then assigned to activation function ho is the nonlinear processing unit, to help in learning abstractions and embeds non-linearity in the feature space. This way it generates different patterns helps in learning of semantic differences in images [7]. Until now this algorithm has shown an amazing performance in images classification, detection, segmentation, and extracting features.

\subsection{Data augmentation}

Data Augmentation are methods that used to expand the size of dataset by create one or multiple new data slightly modified of each existing data, the purpose of these techniques is to assist machine learning algorithms to reducing the over fitting. In our case we use 9 copies for each face images [12].

\subsection{Binary classification}

In deep learning, binary classification is the create a model with two outputs able to predict true or false from input data that are divided into two groups, in generally it can solve each problem with average double classification issues, since the output is simple, the accuracy of these algorithms are higher than the other classifiers and it's easy for the model to predict results [13].

\subsection{Multi-label learning}

One of the major problems of classification that Deep learning try to model is to assign more than two classes to multiple output, this technic is called the multi-label classification. Multi-label classification is how to find a model that can map inputs to binary vectors, this technic is a generalization of binary and multiclass classification we assign a value 0 or 1 for each element in output [14].

\section{RELATED WORK}

Mao et al., propose a new algorithm to deal with the face attribute extraction from facial images, the algorithm called deep multi-task multi-label CNN (DMM-CNN), by dividing the facial attributes in two categories objective and subjective they manage to run two different network architectures taking advantage of multitask learning, and adopting dynamic weighting scheme to resolve the problem of diverse learning complexities [8]. In the same field Ehrlich and Shields propose there multitask learning facial attributes approach [15], while Mandel, Pascanu propose a method based on shared features between these attributes, by using multi-task restricted boltzmann machine (MT-RBM) [16], they were able to learning a joint feature representation from facial landmark points for all attributes, following by approach subsist of a bottomup/top-down pass for learning the shared representation of multitask models, and bottom-up pass for prediction of tasks. This approach has reached some good result by average of $87 \%$ accuracy for all attributes on CelebA dataset [9]. 
Zhuang et al. present a novel for multi-label learning facial attributes using deep transfer neural network method named face multi-label transfer network (FMTNet), as it names this method reside in 3 major networks, face detection, multi-label learning and transfer learning network, the second network consist of predicting multiple facial attributes simultaneously, to increase performance, and reduces feature redundancy with the proposed loss weight scheme, the third network is the unsupervised learning, for the adaptation of unlabeled facial attribute classification, their method reaches an average $84.34 \%$ accuracy on LFWA dataset [9].

H. Ding et al. present novel to improve attribute classification, true cascade network learns the locating of the face region and performs attribute classification without alignment, i) The network is designed to automatically detect specific regions of attributes; ii) a construction and combination of a hole imagebased network and a multiple part-based network by the region switch layer for final attribute classification [17]. Khan et al. propose a framework focusing on three human aspects the gender, race and age, the framework use two CNN, the first as segmentation model use to parses a face into seven dance classes than create a probability maps for each face class, the second model is to extract features from probability maps of the corresponding class for each three aspect mentioned before [18].

\section{IMPLEMENTATION OF THE METHOD}

Our approach uses both high prediction rate for binary classification models and simplified images into sketch, for extracting some features with more visible attributes. Considering our goal, we develop an algorithm that consists first to detect the face on training data, then it transforms our input data to sketch. Next, it splits, for each attribute, the preprocessed data into 1 (folder 1 ) if the attribute exists or 0 (folder 0 ) if not. After that, we augmented our data for more accurate training, we feed two networks, one for real images and another for sketch images as shown in Figure 1. As training data, we use CELEBA [19] attribute datasets with more than 200000 images, each with 40 attributes. At last, we generate images from the model StyleGAN and we apply upon it our method to create a new dataset able to improve our training and open the door for our next research.

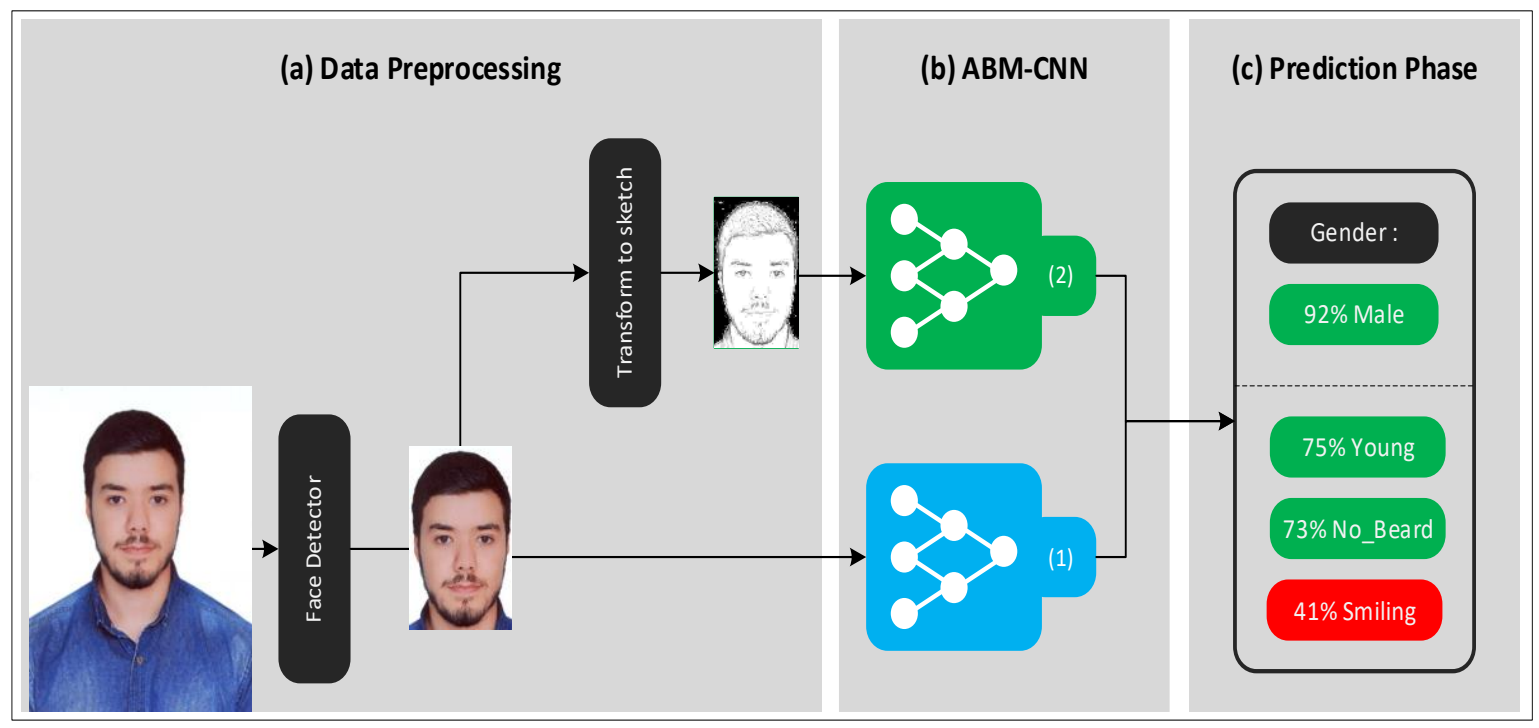

Figure 1. ABM-CNN training model

\subsection{CNN architecture}

We initialize the parameters for our CNN with a batch size with 32 height and width with 180, so the input layer has $180 * 180 * 3$ neurons, with 10 intermediate hidden layers, 3 Convolution2D layers (16, 32, 64), 3 Max_Pooling2D layers (16, 32, 64), 1 Dropout layer (64), 1 Flatten layer (30976), 2 Dense layers $(128,2)$, and 2 output neurons for the binary classification as shown in Figure 2. Our model was trained between 15 and 20 epochs, all the data available is passed through the neural network 15 with some attribute and 20 times with others. We use Relu as activation function, while the optimizer function was 'Adam', and we used Accuracy as metrics. We train over 4 million parameters. 


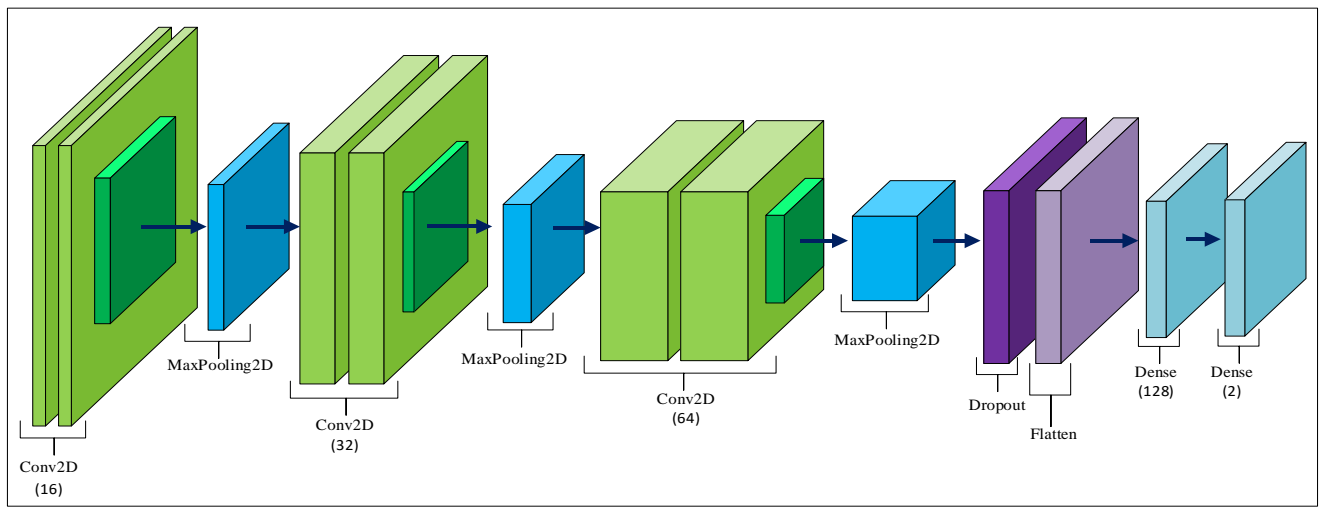

Figure 2. CNN model layers

\subsection{Step of our work}

- First phase: Prepare our data; Split our dataset into 40 folders. Every folder contains subfolder attributes and opposite attributes, and even every subfolder contains three subfolders: trains, validation and tests. We distribute our data in the following form: 70\% for the train folder, and 15\% for each validation and test.

- Second phase: Transform data; for running algorithms on both real human face photos and sketch ones, unfortunately the dataset available of sketch photos is limited. For that reason, we are going to transform CELEBA to sketch.

- Third phase: Dataset preprocessing; First of all, apply the augmented function on datasets, read all images in our train folder and transform-it to matrices and vectors to train our model to reach the goal of the best performance.

- Fourth Phase: Build our models; once we finish training our models, we save them. Every model is fit on a training dataset, than the test dataset is used to validate the accuracy of our final model.

- Fifth Phase: The Evaluation of our final model quality by predicting outside data and compare the results.

- Sixth Phase: We combine the predict result on both real image and sketch image by calculating best accuracy for each attribute.

- Seventh Phase: Create new dataset that contains generated facial images by Style-GAN. We also generate a CSV file for attribute classification results. This will be done in future work.

\subsection{Hardware characteristics}

To test our DL-model, we use the remotely accessible high-performance computing (HPC) infrastructure Cluster HPC-MARWAN [20]:

- Compute Nodes: $2 *$ Intel Xeon Gold 6148(2.4GHz/20-core) / 192 GB RAM

- GPU Node : $2 *$ NVIDIA Tesla P100 / 192 GB RAM

- Storage Node: $2 *$ Intel Xeon Silver 4114(2.2GHz/20-core $) / 18 *$ SATA 6 TB

\section{RESULT AND DISCUSSION}

We start our experience with training and comparing residual networks using on CelebA dataset. After the implementation of ABM-CNN algorithm, we evaluate each neural network on every facial attribute, by their efficiency using accuracy, loss, validation accuracy, validation training, epochs and training time. We conducted the experiment with $70 \%$ of our dataset for training, $15 \%$ for testing and the same rate for validation, a batch size of 32, and a number of epochs between 15 and 20. For all attributes, we have almost 4 million trainable parameters. As shown in Table 1, the results of our approach are promising. We notice a better performance, an average of all attributes reaching $90.05 \%$ accuracy with real images and $88.93 \%$ accuracy with sketch, which gives us a very good prediction result. We see some attributes exceed the $96 \%$ accuracy marks such as (gender, baldness, eyeglass, pale skin, hair color), while the others do not go down to $74.61 \%$ accuracy; this percentage returns to the oval face attribute in sketch, while the average duration of an epoch is $10 \mathrm{~min}$, or almost $4 \mathrm{~h}$ for 20 epochs with $140 \mathrm{~ms}$ / step. The result of training real image is better than sketch for most attributes. However, in Figure 3 we notice in some attributes training neural with sketch that we can predict better results for the following attribute (bald, big lips, double chin, eyeglasses, no beard). We conclude that we can sketch models to predict these attributes instead of real images models. Our accuracy jumps from $90.4 \%$ to $90.54 \%$, a such rate gives more precisely prediction than the normal models. 
Table 1. Results of training ABM-CNN algorithm on every facial attribute, both with celebA dataset Dataset CelebA Real Images CelebaA Sketch Images

\begin{tabular}{|c|c|c|c|c|c|c|c|c|c|c|}
\hline Attributes & 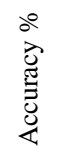 & $\begin{array}{l}0 \\
0 \\
0 \\
0\end{array}$ & 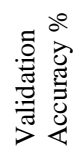 & 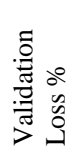 & 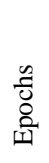 & 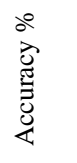 & $\begin{array}{l}0 \\
0 \\
0 \\
0 \\
1\end{array}$ & 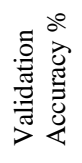 & 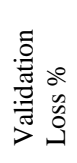 & $\begin{array}{l}\frac{n}{0} \\
\text { षे } \\
\text { जी }\end{array}$ \\
\hline 1_No_Beard_ & 94.7 & 13.1 & 95 & 12.1 & 20 & 95.4 & 11.9 & 95.8 & 10.9 & 20 \\
\hline 2_Wavy Hair_ & 86.4 & 37 & 83.6 & 36.8 & 20 & 81.1 & 40.8 & 82 & 39.7 & 20 \\
\hline 3_5o'clockShadow_ & 94 & 17 & 94.1 & 17.1 & 15 & 91.8 & 18.7 & 92.1 & 18.2 & 20 \\
\hline 4_Attractive_ & 81 & 41 & 80 & 42 & 15 & 80.3 & 41.1 & 80.5 & 41.2 & 20 \\
\hline 5_Brown_Hair_ & 86.5 & 30 & 87 & 30.5 & 15 & 81.3 & 39.8 & 81 & 40.8 & 20 \\
\hline 6_Bags_Ūder_ĒEe_ & 83.5 & 35 & 83 & 38 & 15 & 83.2 & 36.2 & 83.1 & 36.45 & 20 \\
\hline 7_Sideburns_ & 98.6 & 7.8 & 96.5 & 7.8 & 20 & 96.6 & 8.3 & 96.7 & 7.8 & 20 \\
\hline 8_Big_Lips_ & 77.4 & 48.2 & 77.7 & 47.5 & 15 & 81.9 & 38.9 & 81.7 & 0.39 & 20 \\
\hline 9_Big_Nose_ & 83 & 38.5 & 83.1 & 38 & 15 & 77.2 & 48.9 & 77.2 & 49.2 & 20 \\
\hline 10_Arched_Eyebrow_ & 83 & 36 & 83.8 & 35.2 & 15 & 82.9 & 35.9 & 83.4 & 35 & 20 \\
\hline 11_Blond_Hair_ & 96 & 12.8 & 96.1 & 12.5 & 15 & 93.6 & 15.5 & 93.8 & 15.5 & 20 \\
\hline 12_Blurry_ & 95.8 & 11 & 96.5 & 10.2 & 15 & 95.7 & 11.7 & 95.3 & 18.2 & 20 \\
\hline 13_Bushy_Eyebrow_ & 92 & 21.8 & 90.8 & 22.1 & 15 & 90.5 & 23 & 90.9 & 22 & 20 \\
\hline 14_Chubby_ & 95 & 13.5 & 95.1 & 12.8 & 15 & 94 & 13.9 & 95 & 13.3 & 20 \\
\hline 15_Eyeglasses_ & 99.5 & 2.2 & 99.5 & 2 & 15 & 99.6 & 2.7 & 99.5 & 2.7 & 20 \\
\hline 16_Mouth_Open_ & 93 & 19.5 & 93.5 & 18.5 & 15 & 90.2 & 23.7 & 90.4 & 23.9 & 20 \\
\hline 17_Mustache_ & $\mathbf{9 7 . 2}$ & 9 & 98.1 & 8.7 & 15 & 96.1 & 9.7 & 96.1 & 9.3 & 20 \\
\hline 18_Narrow Eyes_ & 89.8 & 26 & 90 & 25.4 & 15 & 89.8 & 26.3 & 89.8 & 26.4 & 20 \\
\hline 19_Oval_Face_ & 76.8 & 52.5 & 75.6 & 51.6 & 20 & 74.6 & 52.7 & 75 & 51.9 & 20 \\
\hline 20_Pale_Skin_ & 98.3 & 8.7 & 96.7 & 8.8 & 20 & 95.8 & 15.5 & 95.7 & 16.4 & 20 \\
\hline 21_Double_Chin_ & 95.9 & 11 & 96 & 10.5 & 15 & 96 & 11 & 96.1 & 10.5 & 20 \\
\hline 22_Pointy_Nose_ & 77 & 48.9 & 75.8 & 0.49 & 20 & 75.5 & 49.7 & 75.7 & 49.7 & 20 \\
\hline 23_Recede Hair_- & 93.9 & 15.7 & 93.5 & 15.3 & 20 & 92.8 & 16.9 & 93 & 16.2 & 20 \\
\hline 24_Rosy Cheeks_ & 94.9 & 12.1 & 95 & 12.2 & 20 & 94.2 & 14.2 & 93.8 & 14.79 & 20 \\
\hline 25_Smiling_ & 93.8 & 19.7 & 91.9 & 19.5 & 20 & 90.8 & 21.6 & 91.4 & 20.5 & 20 \\
\hline 26_Bald_ & 99 & 3.5 & 98.6 & 3.2 & 15 & 99.4 & 4 & 99.3 & 4.1 & 20 \\
\hline 27_Straight Hair_ & 85 & 39.3 & 81.8 & 39.5 & 20 & 80.9 & 41 & 81.2 & 40.7 & 20 \\
\hline Average & 90.4 & 23.36 & 89.94 & 21.42 & & 88.93 & 24.95 & 89.09 & 23.55 & \\
\hline
\end{tabular}

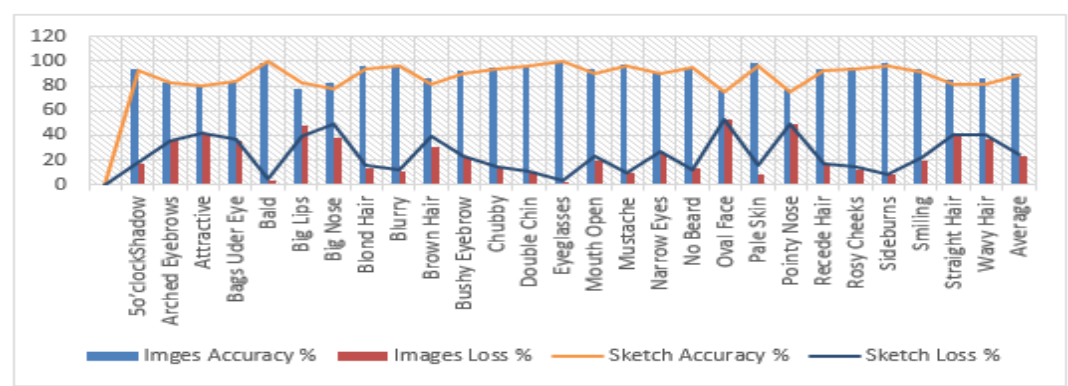

Figure 3. Comparison of Loss and Accuracy for every attribute

\subsection{Comparison ABM-CNN with FAC existing methods}

In this section, we compare the performance of our proposed method ABM-CNN with various FAC methods, like Panda [21], this method use part based models to extract features and SVM [22] as classifiers, like LNets+ANet which use it to for each attribute, besides two localization networks and one attribute network. MOON [23] an approach which uses a combination of optimization methods resolving the issues of imbalance problem for multi-label, as NSA [24] an approach uses the segment-based methods for FAC, MCNN-AUX [25] in another hand based on attribute location which divides them into nine groups, last one the MCFA [26] method which exploits the inherent dependencies between FAC and auxiliary tasks (face detection and FLD). As shown in Table 2 ABM-CNN surmount the FAC methods we just mentioned with an average accuracy of $90.54 \%$ of all attributes on CelebA specially in certain attributes (Bald, Big_Lips, Blond_Hair, Mustache, Narrow_Eyes, Pale_Skin, Sideburns, Smiling, Wavy_Hair) which overpass all the FAC methods, however, in some attribute ABM-CNN fails to obtain a high rate as his competitor (Bags_Under_Eye, Big_Nose, No_Beard, Rosy_Cheeks). On the whole, the performance comparison between all the competing methods shows the effectiveness of the proposed method. The binary classification method has proven her high score prediction over multi-class or multi-lable used by related work, in FAC methods. 
Table 2. Comparison with state-of-the-art FAC methods

\begin{tabular}{|c|c|c|c|c|c|c|c|}
\hline \multirow{2}{*}{$\begin{array}{l}\text { Dataset } \\
\text { Models }\end{array}$} & \multicolumn{7}{|c|}{ CELEBA } \\
\hline & Panda & LNets+ANet & MOON & NSA & $\begin{array}{c}\text { MCNN- } \\
\text { AUX }\end{array}$ & MCFA & ABM-CNN \\
\hline \multicolumn{8}{|l|}{ Attributes } \\
\hline 1_Eyeglasses & 98.00 & 99.00 & 99.47 & 99.51 & 99.63 & 100.0 & 99.60 \\
\hline 2_Arched- & 78.00 & 79.00 & 82.26 & 82.56 & 83.42 & 83.00 & 83.00 \\
\hline \multicolumn{8}{|l|}{ Eyebrows } \\
\hline 3_Mustache & 93.0 & 95.00 & 96.82 & 95.86 & 96.88 & 97.00 & 97.20 \\
\hline 4_5o'clockShadow & 88.00 & 91.00 & 94.03 & 93.13 & 94.51 & 94.00 & 94.00 \\
\hline 5_Bags_Under_Eye & 79.00 & 79.00 & 84.92 & 84.86 & 84.92 & 85.00 & 83.50 \\
\hline 6_Pointy_Nose & 71.00 & 72.00 & 76.46 & 76.47 & 77.47 & 77.00 & 77.00 \\
\hline 7_Bald & 96.00 & 98.00 & 98.77 & 98.03 & 98.90 & 99.00 & 99.40 \\
\hline 8_Big_Lips & 67.00 & 68.00 & 71.48 & 69.28 & 71.47 & 72.00 & 81.90 \\
\hline 9_Big_Nose & 75.00 & 78.00 & 84.00 & 83.81 & 84.53 & 84.00 & 83.00 \\
\hline 10_Blond_Hair & 93.00 & 95.00 & 95.86 & 95.76 & 96.01 & 96.00 & 96.00 \\
\hline 11_Narrow_Eyes & 84.00 & 81.00 & 86.52 & 86.88 & 87.23 & 87.00 & 89.75 \\
\hline 12_Bushy_Eyebrow & 86.00 & 90.00 & 92.62 & 92.66 & 92.84 & 92.00 & 92.00 \\
\hline 13_Attractive & 81.00 & 81.00 & 81.67 & 82.76 & 83.06 & 83.00 & 81.00 \\
\hline 14_Chubby & 86.00 & 91.00 & 95.44 & 94.94 & 95.67 & 96.00 & 95.00 \\
\hline 15_Double_Chin & 88.00 & 92.00 & 96.32 & 95.80 & 96.32 & 96.00 & 96.00 \\
\hline 16_Mouth-Open & 93.00 & 92.00 & 93.54 & 93.78 & 93.74 & 93.00 & 93.00 \\
\hline 17_Sideburns & 93.00 & 96.00 & 97.59 & 97.17 & 97.85 & 98.00 & 98.50 \\
\hline 18_Blurry & 86.00 & 84.00 & 95.67 & 95.96 & 96.17 & 96.00 & 95.80 \\
\hline 19_Oval_Face & 65.00 & 66.00 & 75.73 & 74.93 & 75.84 & 75.00 & 75.61 \\
\hline 20_Pale_Skin & 91.00 & 91.00 & 97.00 & 97.00 & 97.05 & 97.00 & 98.30 \\
\hline 22_No_Beard & 93.00 & 95.00 & 95.58 & 96.17 & 96.05 & 96.00 & 95.40 \\
\hline 23_Brown_Hair & 77.00 & 80.00 & 89.38 & 88.25 & 89.15 & 88.00 & 86.50 \\
\hline 24_Recede_Hair & 85.00 & 89.00 & 93.56 & 92.25 & 93.81 & 94.00 & 93.90 \\
\hline 25_Rosy_Cheeks & 87.00 & 90.00 & 94.82 & 94.79 & 95.16 & 95.00 & 94.40 \\
\hline 26_Straight_Hair & 69.00 & 73.00 & 82.26 & 80.41 & 83.58 & 85.00 & 85.00 \\
\hline 27_Smiling & 92.00 & 92.00 & 92.60 & 92.70 & 92.73 & 93.00 & 93.80 \\
\hline 28_Wavy_Hair & 77.00 & 80.00 & 82.47 & 81.70 & 83.91 & 85.00 & 86.00 \\
\hline Average & 84.00 & 85.64 & 89.59 & 89.29 & 90.03 & 89.96 & 90.54 \\
\hline
\end{tabular}

\subsection{Generate news images and predict attribute for new dataset}

In this part, we try to create a new dataset by generating images from Style-GAN and apply on them ABM-CNN for the extraction of pertinent attributes over $50 \%$ predicted rate and saved-it in CSV file like is shown in Figure 4. We cite few reasons with adapted ABM-CNN to extract features form GAN models [27], i) create dataset of high quality compared to existing datasets and unlimited data; ii) create datasets with different face poses and face transformation; iii) the lack of large databases for portraits; iv) the problem of policy in certain private dataset; v) Research is moving towards the path of data generation and unsupervised learning.

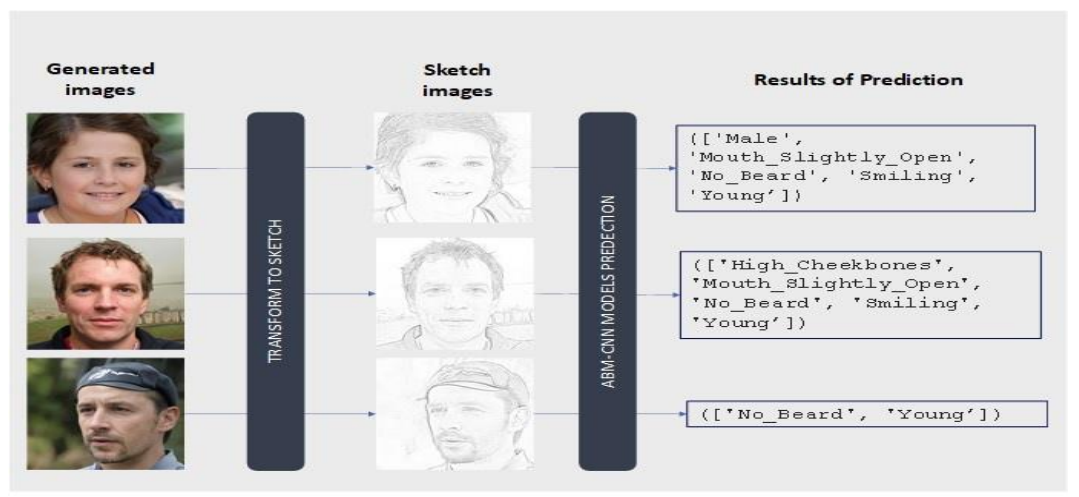

Figure 4. Predict facial attributes from generated images using ABM-CNN

\section{CONCLUSION}

In this paper, we propose a novel framework called Augmented Binary multi-label CNN method (ABM-CNN) to improves the performance of FAC. The main work of our method is handling each attribute as a binary problem "exists or not existing", then transforming the data given in sketch and running two neural networks. The first one is for predicting real images and the second for sketch images. Experiments on 
the public CelebA dataset have demonstrated that ABM-CNN achieves higher performance compared with several related works on FAC methods. Finally, we generate images via Style-GAN and apply on it our approach to resolve some exciting problems that researchers usually face in the field.

\section{REFERENCES}

[1] I. Idrissi, M. Boukabous, M. Azizi, O. Moussaoui, and H. El Fadili, "Toward a deep learning-based intrusion detection system for IoT against botnet attacks," IAES International Journal of Artificial Intelligence IJ-AI, vol. 10, no. 1, pp. 110-120, 2021, doi: 10.11591/ijai.v10.i1.pp110-120.

[2] I. Idrissi, M. Azizi, and O. Moussaoui, "IoT security with Deep Learning-based Intrusion Detection Systems: A systematic literature review," Fourth International Conference on Intelligent Computing in Data Sciences ICDS, 2020, pp. 1-10, doi: 10.1109/ICDS50568.2020.9268713.

[3] H. Han, B. F. Klare, K. Bonnen, and A. K. Jain, "Matching Composite Sketches to Face Photos: A ComponentBased Approach," IEEE Trans. on Information Forensics and Security, vol. 8, no. 1, pp. 191-204, Jan. 2013, doi: 10.1109/TIFS.2012.2228856.

[4] C. D. Frowd, et al., "Catching Even More Offenders with EvoFIT Facial Composites," Third Int. Conference on Emerging Sec. Tech., 2012, pp. 20-26, doi: 10.1109/EST.2012.26.

[5] X. Zheng, Y. Guo, H. Huang, Y. Li, and R. He, "A Survey of Deep Facial Attribute Analysis," International Journal of Computer Vision, vol. 128, no. 8-9, pp. 2002-2034, 2020, doi: 10.1007/s11263-020-01308-z.

[6] M. Boukabous and M. Azizi, "Review of Learning-Based Techniques of Sentiment Analysis for Security Purposes," Innov. in Smart Cities App., vol. 4, pp. 96-109, 2021, doi: doi.org/10.1007/978-3-030-66840-2_8.

[7] A. Khan, A. Sohail, U. Zahoora, and A. S. Qureshi, "A survey of the recent architectures of deep convolutional neural networks," Artificial Intelligence Review Volume, vol. 53, no. 8, 2020, doi: 10.1007/s10462-020-09825-6.

[8] L. Mao, Y. Yan, J. Xue, and H. Wang, "Deep Multi-task Multi-label CNN for Effective Facial Attribute Classification," IEEE Transactions on Affective Computing, doi: 10.1109/TAFFC.2020.2969189.

[9] N. Zhuang, Y. Yan, S. Chen, H. Wang, and C. Shen, "Multi-label Learning Based Deep Transfer Neural Network for Facial Attribute Classification," Pattern Recognit, vol. 80, pp. 225-240, 2018, doi: 10.1016/j.patcog.2018.03.018.

[10] Y. Wu, T. Hassner, K. Kim, G. Medioni, and P. Natarajan, "Facial Landmark Detection with Tweaked Convolutional Neural Networks," IEEE Trans. on Pattern Analysis and Machine Intelligence, vol. 40, no. 12, pp. 3067-3074, 2018, doi: 10.1109/TPAMI.2017.2787130.

[11] T. Karras, S. Laine, M. Aittala, J. Hellsten, J. Lehtinen, and T. Aila, “Analyzing and Improving the Image Quality of StyleGAN," Proc. of the IEEE/CVF Conference on Computer Vision and Pattern Recognition CVPR, 2020.

[12] C. Shorten and T. M. Khoshgoftaar, "A survey on Image Data Augmentation for Deep Learning," Journal of Big Data, vol. 6, no. 1, pp. 1-48, 2019, doi: 10.1186/s40537-019-0197-0.

[13] Binary classification-Wikipedia. [Online]. Available: https://en.wikipedia.org/wiki/Binary_classification.

[14] A. Buyukcakir, H. Bonab, and F. Can, "A novel online stacked ensemble for multi-label stream classification," International Conference on Information and Knowledge Management, 2018, pp. 1063-1072, doi: 10.1145/3269206.3271774.

[15] M. Ehrlich, T. J. Shields, T. Almaev, and M. R. Amer, "Facial Attributes Classification using Multi-Task Representation Learning," Proc. IEEE Conf. Comp. Vision and Pattern Recognition Workshops, 2016, pp. 47-55.

[16] H. L. Ca, M. Mandel, R. Pascanu, Y. Bengio, and B. U. Ca, "Learning Algorithms for the Classification Restricted Boltzmann Machine," Journal of Machine Learning Research, vol. 13, pp. 643-669, 2012.

[17] H. Ding, H. Zhou, S. K. Zhou, and R. Chellappa, "A Deep Cascade Network for Unaligned Face Attribute Classification," 32nd AAAI Conference Artificial Intelligence, 2018, pp. 6789-6796.

[18] K. Khan, M. Attique, R. U. Khan, I. Syed, and T. S. Chung, "A Multi-Task Framework for Facial Attributes Classification through End-to-End Face Parsing and Deep Convolutional Neural Networks," Sensors, vol. 20, no. 2, doi: $10.3390 / \mathrm{s} 20020328$.

[19] Z. Liu, P. Luo, X. Wang, and X. Tang, "Large-scale CelebFaces Attributes (CelebA) Dataset." [Online]. Available: http://mmlab.ie.cuhk.edu.hk/projects/CelebA.html

[20] High Performance Computing (HPC) \& AMD. [Online]. Available: https://www.marwan.ma/index.php

[21] N. Zhang, M. Paluri, M. Ranzato, T. Darrell, and L. Bourdev, "PANDA: Pose Aligned Networks for Deep Attribute Modeling," Proc.s of the IEEE Conf. on Computer Vision and Pattern Recognition, pp. 1637-1644, 2013.

[22] B. Scholkopf and A. J. Samola, "Smola. A: Learning with Kernels-Support Vector Machines, Regularization, Optimization and Beyond," MIT Press, Cambridge, MA, 2001.

[23] E. M. Rudd, M. Günther, and T. E. Boult, "MOON: A mixed objective optimization network for the recognition of facial attributes,” European Conf. Computer Vision, 2016, pp. 19-35, doi: 10.1007/978-3-319-46454-1_2.

[24] U. Mahbub, S. Sarkar, and R. Chellappa, "Segment-Based Methods for Facial Attribute Detection from Partial Faces," IEEE Trans. Affec. Computing, vol. 11, no. 4, pp. 601-613, 2020, doi: 10.1109/TAFFC.2018.2820048.

[25] E. M. Hand and R. Chellappa, "Attributes for Improved Attributes: A Multi-Task Network for Attribute Classification," arXiv Cornell University, 2016.

[26] N. Zhuang, Y. Yan, S. Chen, and H. Wang, "Multi-task Learning of Cascaded CNN for Facial Attribute Classification," 24th Int. Conf. on Pattern Recognition ICPR, 2018, doi: 10.1109/ICPR.2018.8545271.

[27] M. Berrahal and M. Azizi, "Review of DL-Based Generation Techniques of Augmented Images using Portraits Specification," Fourth Int. Conf. on Intelligent Computing in Data Sciences ICDS, 2020, pp. 1-8, doi: 10.1109/ICDS50568.2020.9268710. 